\title{
Clinical and Quality of Life Outcomes of a Combined Synthetic Scaffold and Autogenous Tissue Graft Procedure for Articular Cartilage Repair in the Knee
}

Fernando Martins Rosa ( $\nabla$ nandomrx@hotmail.com )

Universidade Federal do Parana https://orcid.org/0000-0002-7373-9098

Julio Cesar Fernandes

Universite de Montreal

Josée Delisle

Hôpital du Sacré-Coeur de Montréal: Hopital du Sacre-Coeur de Montreal

Pierre Ranger

Hôpital du Sacré-Coeur de Montréal: Hopital du Sacre-Coeur de Montreal

Mauro Batista Albano

Universidade Federal do Parana

Edmar Stieven Filho

Universidade Federal do Parana

\section{Research Article}

Keywords: Cartilage, Tissues, Support, Cartilage, Hyaline, Orthopedics, Arthroplasty, Subchondral, Knee Surgery, Sports Surgery, Scaffold

Posted Date: September 23rd, 2021

DOl: https://doi.org/10.21203/rs.3.rs-917430/v1

License: (c) (i) This work is licensed under a Creative Commons Attribution 4.0 International License. Read Full License

Version of Record: A version of this preprint was published at Journal of Orthopaedic Surgery and Research on February 20th, 2022. See the published version at https://doi.org/10.1186/s13018-02203010-x. 


\section{Abstract}

Background: Injuries to the articular cartilage of the knee often fail to heal properly, due to the hypocellular and avascular nature of this tissue. Subsequent disability can limit participation in sports and decrease quality of life. Subchondral bone perforations are used for the treatment of small defects. Larger lesions filling out the central portion becomes difficult, and scaffolds can be used as adjuvants, providing a matrix onto which the defect can be filled in completely and also autogenous cartilage grafts can be combined, acting as an inducer and improving healing quality, all in a single procedure.

Methods: Evaluate the clinical and quality of life outcomes of patients with articular cartilage lesions of the knee undergoing repair via a microfracture technique combined with a synthetic scaffold and autogenous cartilage graft, with transosseous sutures and fibrin glue fixation, at 12 months of follow-up. Secondarily, assess whether combined procedures, previous surgical intervention, traumatic aetiology, lesion location, and age affect outcomes. Observational study of adult patients (age 18-66 years) with symptoms consistent with chondral or osteochondral lesions, isolated or multiple, ICRS grade III/IV, 2-12 $\mathrm{cm}^{2}$ in size. Patients with corrected angular deviations or instabilities were included. Those with $\mathrm{BMI}>40$ $\mathrm{kg} / \mathrm{m}^{2}$, prior total or subtotal ( $>30 \%$ ) meniscectomy, second-look procedures, and follow-up $<6$ months were excluded. Pain (VAS), physical activity (IKDC), osteoarthritis (WOMAC), and general quality of life (SF-36) were assessed.

Results: 64 procedures were included, comprising 60 patients. There was significant improvement $(P<0.05)$ in VAS (5.92 to 2.37), IKDC score (33.44 to 56.33), and modified WOMAC score (53.26 to 75.93$)$ after surgery. The SF-36 showed significant improvements in the physical and mental domains (30.49 to 40.23 and 46.43 to 49.84 respectively; both $P<0.05$ ).

Conclusions: Combination of microfractures, autogenous crushed cartilage graft, synthetic scaffold, and transosseous sutures with fibrin glue provides secure fixation for treatment of articular cartilage lesions of the knee. At 12-month follow-up, function had improved by 20 points on the IKDC and WOMAC, and quality of life by 10 points on the SF-36. Age $>45$ years had a negative impact on outcomes.

\section{Background}

Injuries to the articular cartilage of the knee often fail to heal properly, due to the hypocellular and avascular nature of this tissue. Subsequent disability can limit participation in sports and decrease quality of life, making surgical treatment of these lesions an attractive option [1]. In a meta-analysis, surgical repair of articular cartilage lesions was associated with a $76 \%$ rate of return to sport. Mediumterm activity scores for this procedure were comparable to those achieved with meniscus repair [2].

The subchondral bone perforations introduced by Pridie [3] and modified by Steadman et al. [4] are still used for the treatment of small $\left(2\right.$ to $\left.3 \mathrm{~cm}^{2}\right)$, ICRS grade III and IV chondral defects. These can repair the defect from the periphery towards the center, but in lesions larger than $3 \mathrm{~cm}^{2}$, filling out the central portion becomes difficult. To improve this, scaffolds can be used as adjuvants, providing a matrix onto which the 
defect can be filled in completely. Autogenous cartilage grafts can be combined with this matrix, acting as an inducer and improving healing quality, all in a single procedure [5].

Studies have reported the impact of surgical cartilage repair on physical activity scores (IKDC), osteoarthritis scales (WOMAC), and visual analogue scales (VAS) of pain; however, few have assessed this impact on quality of life outcomes measured with validated instruments, such as the SF-36. A brief review of the literature found no studies using the WOMAC, IKDC, and SF-36 concurrently.

Thus, the objective of this study is to evaluate the clinical and quality of life outcomes of the microfracture technique, combined with autologous cartilage graft and a synthetic matrix, in the repair of articular cartilage lesions in the knee.

\section{Methods}

This was a retrospective analysis of medical records of patients who underwent surgical repair of articular cartilage lesions at a single hospital. Approval was obtained from the Ethics Committee of University of Montreal (dossier no. CER 2021-2115).

The inclusion criteria were: patients aged 18 to 66 years, with symptoms consistent with single or multiple chondral or osteochondral lesions, grade III/IV on the International Cartilage Repair Society (ICRS), classification, 2 to $12 \mathrm{~cm}^{2}$ in size.

The exclusion criteria were: $\mathrm{BMI}>40 \mathrm{~kg} / \mathrm{m}^{2}$, meniscectomy $>30 \%$ in the treated compartment, secondlook procedures, and duration of follow-up 6 months or less.

Patients with axis deviation of the lower limbs, patellofemoral joint abnormalities, and ligament instability were accepted. Any such issues were corrected during the same surgical procedure.

Data were collected pre-operatively and at 6 and 12 months post-operatively. All patients completed a subjective Visual Analogue Scale (VAS) for pain; the transformed WOMAC score and International Knee Documentation Committee (IKDC) for functional assessment; and the SF-36 instrument for quality of life.

Patients were divided into two groups for comparison, in order to ascertain whether there was any statistically significant difference in the following variables at 12 months versus baseline: combination with another intervention during the same procedure; history of previous surgery; aetiology (traumatic or non-traumatic); lesion location (patellofemoral or femorotibial); and age (older or younger than 45 years).

Failure was defined as implant loosening, absence of improvement within 12 months, or need for a second procedure.

The scaffold used was a synthetic, single-phase, sterile, acellular, absorbable sponge-type membrane, 1.8 $\mathrm{mm}$ thick, composed of hyaluronic acid and poly-glycolic acid (Chondrotissue ${ }^{\circledR}$, Biotissue AG, Zürich, Switzerland). 


\section{Surgical procedure}

Patients were placed in the supine position with the knee at $90^{\circ}$. No tourniquet was used. Arthroscopy was performed to confirm the size and severity of the cartilage lesion, followed by a mini-arthrotomy depending on the location of the lesion.

The subchondral bone was exposed until a 90-degree well-defined border between the cartilage and the bone was obtained. Microfractures were performed from the periphery towards the centre, with a diameter of $1.2 \mathrm{~mm}$ and a distance of 3 to $4 \mathrm{~mm}$ between drill holes.

A piece of disposable foil was placed on the bed of the lesion to mark out its shape. This was then used as a mould for the membrane, which was cut to fit the defect.

The scaffold was prepared with sutures (\#1 absorbable poly-glycolic acid) at each end of the membrane. The suture knots were pre-formed so they would remain buried in the bone. The scaffold was hydrated with the patient's own blood (Fig. 1).

Samples of autogenous cartilage from the healthy intercondylar area were harvested with a trephine and crushed with a scalpel blade. The resulting autogenous cartilage graft was placed in the bed of the lesion, followed by the scaffold, and the membrane was secured with buried transosseous sutures. After membrane fixation, fibrin glue was applied for added final stability (Fig. 2).

No orthotic devices or splints were used post-operatively. In cases where any additional procedures were necessary, such as osteotomies or repair of ligament injuries, these were performed before the articular surface was repaired.

Rehabilitation was started on the first postoperative day, with range-of-motion exercises. Patients were cleared for progressive weight-bearing immediately in case of patellofemoral lesions and after the sixth postoperative week for femoral condyle lesions. Activities were resumed 12 weeks after the operation, and gradual return to sport was allowed after 6 months.

\section{Statistical analysis}

Patients were evaluated pre-operatively and at 6 and 12 months post-operatively. Analysis of covariance (ANCOVA) was used for comparison between variables. P-values $<0.05$ were deemed statistically significant.

\section{Results}

Sixty patients were evaluated ( 36 women and 24 men, mean age $44 \pm 12.7$ years), for a total of 64 knees (33 right and 31 left).

Overall, 75 articular cartilage lesions were treated (25.3\% grade III and $74.6 \%$ grade IV). Eleven knees had two lesions each. The average lesion size was $4.4 \pm 2 \mathrm{~cm}^{2}$; the smallest lesion was $2 \mathrm{~cm}^{2}$, and the largest, 
$12 \mathrm{~cm}^{2}$. The patella was the most frequent site of injury, accounting for $39.1 \%$ of the cases, followed by the femoral condyles with $37.5 \%$. The remainder were combined lesions.

When a concomitant procedure was combined with articular surface repair, osteotomy of the anterior tibial tuberosity (ATT) was the most common intervention, prevalent in $23.4 \%$ of cases (always performed to correct patellar alignment); $14.1 \%$ had a partial meniscectomy, $14.1 \%$ had a tibial valgus osteotomy, and $18.7 \%$ had no additional procedures.

Just over half of procedures (51.6\%) were performed on patients with progressive lesions and no preceding trauma, while the remaining $48.4 \%$ had a history of trauma. In $71.9 \%$ of cases, there had been no previous surgery.

Regarding pain improvement, VAS scores decreased from $5.92( \pm 2.2)$ to $2.37( \pm 1.9) \mathrm{cm}(P<0.001)$ at 12 months postoperatively.

IKDC scores were consistent with progressive functional improvement, as shown in Fig. 3.

All subjective WOMAC scores (pain, joint stiffness, and functional limitation) improved significantly from baseline after surgery $(P<0.001)$. Details are given in Fig. 4 .

Improvement in the physical component of the SF-36 quality of life score is shown in Fig. 5.

The mental component of the SF-36 score for quality of life demonstrated improvement $(P<0.05)$, rising from 46.43 at baseline to 49.84 at 12 months.

When stratified into groups, age was the only relevant factor, with more favourable outcomes in patients under age 45 on the IKDC score (from 37.8 to 65.0 in those younger than 45 , versus 30.3 to 50.0 in those aged 45 or older; $P=0.010$ ) and total WOMAC score (from 60.8 to 84.4 for those younger than 45 , versus 48.4 to 69.7 for those 45 or older; $P=0.022$ ). Better results were also achieved by patients under 45 on the VAS and SF-36 (physical and mental domains), but in these instruments, the difference was not statistically significant.

Likewise, there was no significant difference in IKDC, total WOMAC, VAS, or SF-36 (physical and mental) scores when comparing patients with traumatic versus non-traumatic aetiology, previous surgery or no previous surgery, articular cartilage repair alone versus combined surgical procedures, or lesion location (patellofemoral versus femorotibial).

In five cases (7.7\%), the procedure was considered to have failed. In one case, the implant came loose from the patella; it was removed and the lesions (multifocal) were debrided. In two other cases, the patients subsequently required total knee replacement (one had a multifocal lesion, and the other, a $9 \mathrm{~cm}^{2}$ lesion). Two cases required second-look patellofemoral arthroplasty (both had multifocal lesions and had undergone combined procedures). 
There were four complications during the follow-up period (6\%), all in successful procedures: one deep venous thrombosis, resolved with conservative treatment only; two cases of arthrofibrosis, which improved with manipulation under anaesthesia; and a superficial skin infection treated with oral antibiotics.

\section{Discussion}

The objective of articular cartilage repair is to provide long-lasting improvement of the patient's quality of life. It is also important that the technique be readily available and as harmless as possible. Cultured chondrocyte implantation provides good repair capacity, but has disadvantages such as high cost, limited availability, and the need for two procedures. Salzmann et al. [5] demonstrated a procedure using a membrane seeded with autologous cartilage cells, which can solve the problems of cost and availability and be performed in a single stage.

Among the 64 repairs carried out in our sample, only once did the membrane actually come loose (1.5\%); the other four cases of failure were likely due to progression of the underlying degenerative process, requiring conversion to arthroplasty (6.25\%). Gomoll et al. [6], in a study of 101 patients with up to 1 year of follow-up, used fibrin glue and sutures along the periphery only when they felt the need for greater fixation, achieving a $5 \%$ reoperation rate due to hypertrophy or delamination.

In our study, VAS scores for pain decreased from 5.92 to 2.37 in the postoperative period $(P<0.001)$, demonstrating clinical improvement in this aspect at 12 months of follow-up. Siclari et al. [7], using the same microfracture scaffold combined with platelet-rich plasma and Smart Nails® (ConMed Linvatec Italy, Milan, Italy) and fibrin glue for fixation, also reported improvement on the KOOS pain scale (from 54.1 to 93.5 ). Verdonk et al. [8] used the MaioRegen ${ }^{\circledR}$ three-layer scaffold (Fin-Ceramica Faenza SpA, Faenza, Italy) and likewise reported a reduction in VAS pain scores, from 63.1 to 22.7. Although pain may not be related to the articular cartilage lesion per se, it is interesting to note that it improves markedly after cartilage repair procedures are performed. This effect can be credited to restoration of the load-bearing property, to reduction of subchondral pressure by the microfractures, or to neovascularization of the lesion site, reducing inflammatory cytokine levels and thus relieving symptoms $[9,10]$.

We also observed improvements in IKDC score at 12 months postoperatively (from 33.44 to $56.33, P<$ 0.001). Berruto et al. [11], using a three-layer matrix implanted through the "press-fit" technique in 49 patients, reported an increase in IKDC score from 45.55 to 70.86 at 12 months. Delcogliano et al. [12] used the same technique and matrix in 19 patients (21 lesions) and demonstrated an approximate gain of 30 score points ( 35.7 to 67.7 ) in 12 months. Theoretically, a three-layer matrix mimics hyaline cartilage more closely. Even so, the clinical outcomes achieved are very similar, and may be attributable to the use of bone perforations or autogenous grafts. In the present study, an approximate 23-point improvement in the mean IKDC score was observed, noting that patients had lower baseline (pre-operative) scores than in the case series of Berruto et al. [11] (45 points) and similar to those reported by Delcogliano et al. [12] 
(35.7 points). This may be attributed to the more advanced average age of our patients compared to the other series (44 years, versus 37 and 33 years respectively) $[11,12]$.

In our cohort, the total WOMAC score improved from 53.26 to $75.93(P<0.001)$ at 12 months postoperatively. Dhollander et al. [13] used allogeneic chondrocyte cell cultures from fresh tissues protected with periosteum. This procedure was performed on 21 patients aged 12 to 47 years, who obtained an average $42 \%$ improvement in WOMAC. For comparison, in our study, we observed a $42.6 \%$ increase in total WOMAC scores. Although part of the cases in the present study were of focal, trauma-related lesions, a higher percentage of patients had progressive pain, probably indicative of osteoarthritis rather than traumatic aetiology; the WOMAC score could be a better tool to assess these patients, since it is a scale designed for degenerative processes.

Few studies have demonstrated improvement in quality of life after cartilage repair. The SF-36 showed an improvement in its physical domain, from 30.49 at baseline to 40.23 post-operatively $(P<0.001)$. Gelber et al. [14] reported an improvement in SF-36 scores from 53.9 to 86.6. In our series, improvement was not as marked, which again may be explained by the higher average age of our patients ( 44 versus 36 years). Older patients tend to have a lower SF-36 because they experience greater difficulty climbing stairs, kneeling, and in other activities of daily living. Cole et al. [15] reported an improvement in scores from 35.4 to 45.5 in the treatment of osteochondritis-related lesions. This more modest improvement, closer to ours, may be due to their patients having osteochondritis dissecans, which carries a less favourable prognosis. The normal range of the SF-36 physical domain has been described as $49.7 \pm 9.4$ [16] in the Canadian population. Thus, it is clear that, despite improvement in patient quality of life, they are unlikely to reach the same level of quality of life experienced by the general population.

Knees with a cartilage lesion but no history of surgery and no comorbid pathologies, so-called "green knees", exclude those most likely to have an unfavourable outcome. However, most patients $(70 \%$ of cases) have combined lesions, or "red knees" $[17,18]$. Martinčič et al. [19] followed 151 patients for an average of 10 years after knee cartilage repair. They found no statistically significant difference in outcomes between patients with and without a history of prior surgery. In our study, we chose not to exclude "red knees", so as to ensure that our sample was representative of the patients most commonly seen in clinical practice. We found no significant differences when patients were stratified by traumatic versus non-traumatic aetiology, prior surgery, combined surgery, or lesion location.

Despite showing an improvement in scores, our patients aged $>45$ years fared rather poorly compared to their younger counterparts. Turinetto et al. [20] demonstrated that advancing age decreases the potential for differentiation, immunomodulatory effect, and migration ability of pluripotential cells and chondrocytes, thus reducing the healing power of tissues. An alternative to improve this capacity would be to introduce cell growth factors such as SDF-1 alpha [21], which has been demonstrated to increase recruitment of cartilage progenitor cells in laboratory studies.

Overall there were five cases of treatment failure (7.7\%). Assessment showed that all patients needing conversion to total knee replacement had lesions that required greater healing potential and were over 45 
years of age. The only patient whose implant came loose was also > 45 years old and had a large lesion, despite no history of trauma. Compared to those of other series, however, our failure rate was similar. Verdonk et al. [8] reported a 5.3\% failure rate, and Delcogliano et al. [12], 2 out of 19 cases $(10.5 \%)$.

\section{Conclusions}

A combined technique consisting of microfractures, autogenous graft, a synthetic scaffold, transosseous sutures and fibrin glue provides secure fixation for treatment of articular cartilage injuries of the knee. Patients experienced an average of 20 points of improvement on the IKDC and WOMAC scales and 10 points of improvement on the SF-36. Age greater than 45 years had a negative impact on outcomes.

\section{Abbreviations}

\begin{tabular}{ll} 
ANCOVA & analysis of covariance \\
ATT & anterior tibial tuberosity \\
\hline ICRS & International Cartilage Repair Society \\
\hline IKDC & International Knee Documentation Committee \\
VAS & Visual Analogue Scale
\end{tabular}

\section{Declarations}

\section{Ethics approval:}

This study was performed in line with the principles of the Declaration of Helsinki. Approval was granted by the Ethics Committee of University of Montreal (CÉR 2021-2115).

\section{Consent to participate:}

Not applicable.

\section{Consent for publication:}

Not applicable.

\section{Availability of data and material:}


The dataset supporting the conclusions of this article is included within the article.

\section{Competing interests:}

The authors have no relevant financial or non-financial interests to disclose.

\section{Funding:}

The authors did not receive support from any organization for the submitted work.

\section{Authors' contributions:}

JCF supervised the study. PR conceptualized the study. JD conducted the formal analysis and investigation of the data. MBA contributed to the review and editing of the study. ESF supervised the study, read and approved the final manuscript, and JCF read and approved the final manuscript.

\section{Acknowledgements:}

Not applicable.

\section{References}

1. Mow VC, Ratcliffe A, Poole AR. Cartilage and diarthrodial joints as paradigms for hierarchical materials and structures. Biomaterials. 1992;13:67-97.

2. Krych AJ, Pareek A, King AH, Johnson NR, Stuart MJ, Williams RJ III. Return to sport after the surgical management of articular cartilage lesions in the knee: a meta-analysis. Knee Surg Sports Traumatol Arthrosc. 2017;25:3186-96.

3. Pridie KH. A method of resurfacing osteoarthritic knee joints. J Bone Joint Surg Br. 1959;41:618-9.

4. Steadman JR, Rodkey WG, Briggs KK. Microfracture to treat full-thickness chondral defects: surgical technique, rehabilitation, and outcomes. J Knee Surg. 2002;15:170-6.

5. Salzmann GM, Calek AK, Preiss S. Second-generation autologous minced cartilage repair technique. Arthrosc Tech. 2017;30;6:e127-31.

6. Gomoll AH, Probst C, Farr J, Cole BJ, Minas T. Use of a type I/III bilayer collagen membrane decreases reoperation rates for symptomatic hypertrophy after autologous chondrocyte implantation. Am J Sports Med. 2009;37 Suppl 1:20S-3S.

7. Siclari A, Mascaro G, Kaps C, Boux E. A 5-year follow-up after cartilage repair in the knee using a platelet-rich plasma-immersed polymer-based implant. Open Orthop J. 2014;8:346 - 54. 
8. Verdonk P, Dhollander A, Almqvist KF, Verdonk R, Victor J. Treatment of osteochondral lesions in the knee using a cell-free scaffold. Bone Joint J. 2015;97-B:318 - 23.

9. O'Reilly A, Kelly DJ. Role of oxygen as a regulator of stem cell fate during the spontaneous repair of osteochondral defects. J Orthop Res. 2016;34:1026-36.

10. Gomoll AH, Minas T. The quality of healing: articular cartilage. Wound Repair Regen. 2014;22 Suppl $1: 30-8$.

11. Berruto M, Delcogliano M, De Caro F, Carimati G, Uboldi F, Ferrua P, et al. Treatment of large knee osteochondral lesions with a biomimetic scaffold: results of a multicenter study of 49 patients at 2year follow-up. Am J Sports Med. 2014;42:1607-17.

12. Delcogliano M, De Caro F, Scaravella E, Ziveri G, De Biase CF, Marotta D, et al. Use of innovative biomimetic scaffold in the treatment for large osteochondral lesions of the knee. Knee Surg Sports Traumatol Arthrosc. 2014;22:1260-9.

13. Dhollander AA, Huysse WC, Verdonk PC, Verstraete KL, Verdonk R, Verbruggen G, et al. MRI evaluation of a new scaffold-based allogenic chondrocyte implantation for cartilage repair. Eur $\mathrm{J}$ Radiol. 2010;75:72-81.

14. Gelber PE, Batista J, Millan-Billi A, Patthauer L, Vera S, Gomez-Masdeu M, et al. Magnetic resonance evaluation of TruFit ${ }^{\circledR}$ plugs for the treatment of osteochondral lesions of the knee shows the poor characteristics of the repair tissue. Knee. 2014;21:827 - 32.

15. Cole BJ, DeBerardino T, Brewster R, Farr J, Levine DW, Nissen C, et al. Outcomes of autologous chondrocyte implantation in study of the treatment of articular repair (STAR) patients with osteochondritis dissecans. Am J Sports Me. 2012;40:2015-22.

16. Hopman WM, Towheed T, Anastassiades T, Tenenhouse A, Poliquin S, Berger C, et al. Canadian normative data for the SF-36 health survey. Canadian Multicentre Osteoporosis Study Research Group. CMAJ. 2000;163:265 - 71.

17. Mor A, Grijota M, Nørgaard M, Munthe J, Lind M, Déruaz A, et al. Trends in arthroscopy-documented cartilage injuries of the knee and repair procedures among 15-60-year-old patients. Scand J Med Sci Sports. 2015;25:e400-7.

18. Martín AR, Patel JM, Zlotnick HM, Carey JL, Mauck RL. Emerging therapies for cartilage regeneration in currently excluded 'red knee' populations. NPJ Regen Med. 2019;4:12.

19. Martinčič D, Mekač J, Drobnič M. Survival rates of various autologous chondrocyte grafts and concomitant procedures. A prospective single-center study over 18 years. Cell Transplant. 2019;28:1439-44.

20. Turinetto $V$, Vitale $E$, Giachino $C$. Senescence in human mesenchymal stem cells: functional changes and implications in stem cell-based therapy. Int J Mol Sci. 2016;17:1164.

21. Yu Y, Brouillette MJ, Seol D, Zheng H, Buckwalter JA, Martin JA. Use of recombinant human stromal cell-derived factor 1a-loaded fibrin/hyaluronic acid hydrogel networks to achieve functional repair of full-thickness bovine articular cartilage via homing of chondrogenic progenitor cells. Arthritis Rheumatol. 2015;67:1274-85. 
Figures
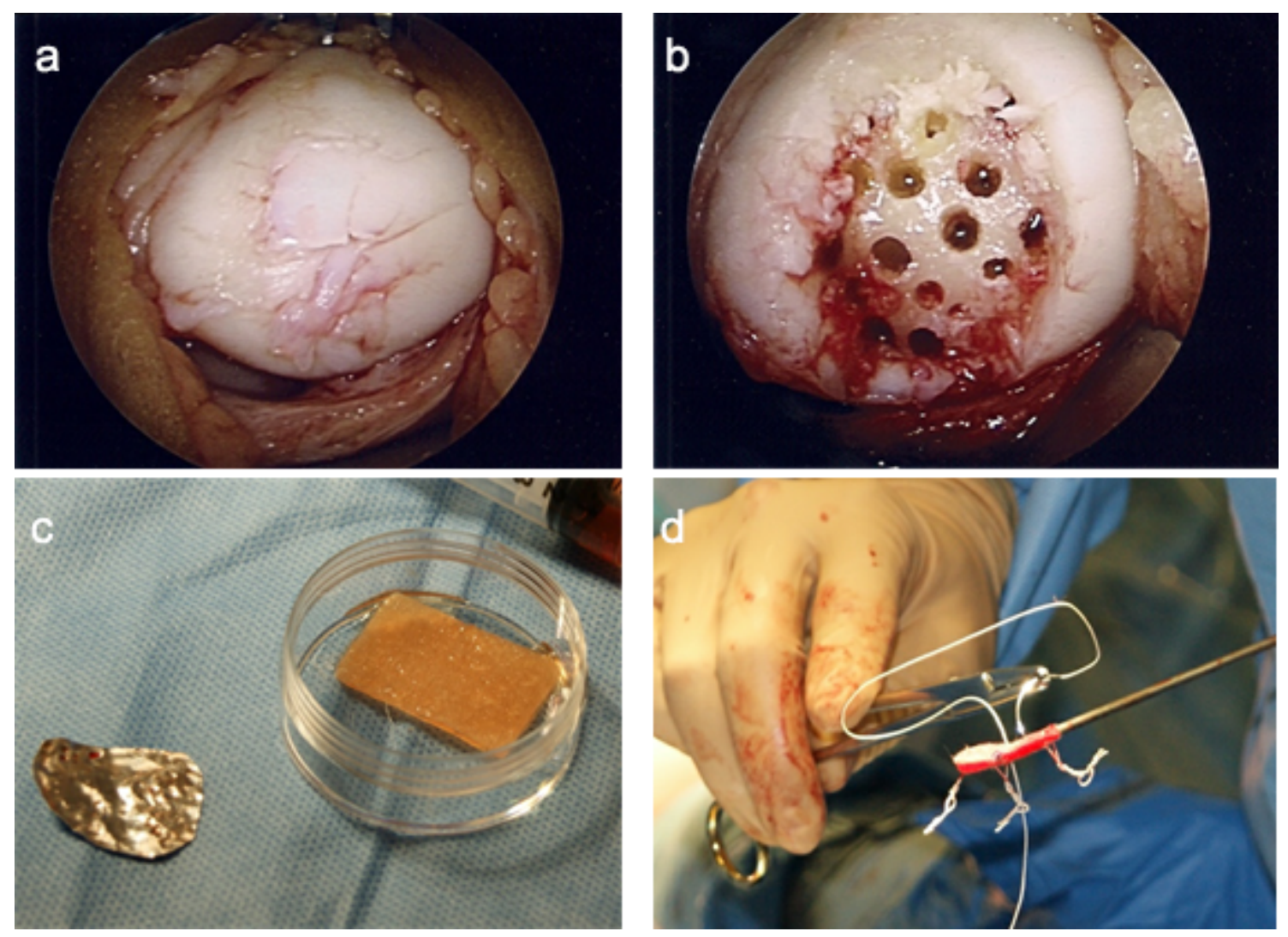

Figure 1

(a) Grade IV patella lesion. (b) Microfractures. (c) Foil mould, cut to shape. (d) Scaffold sutures. 

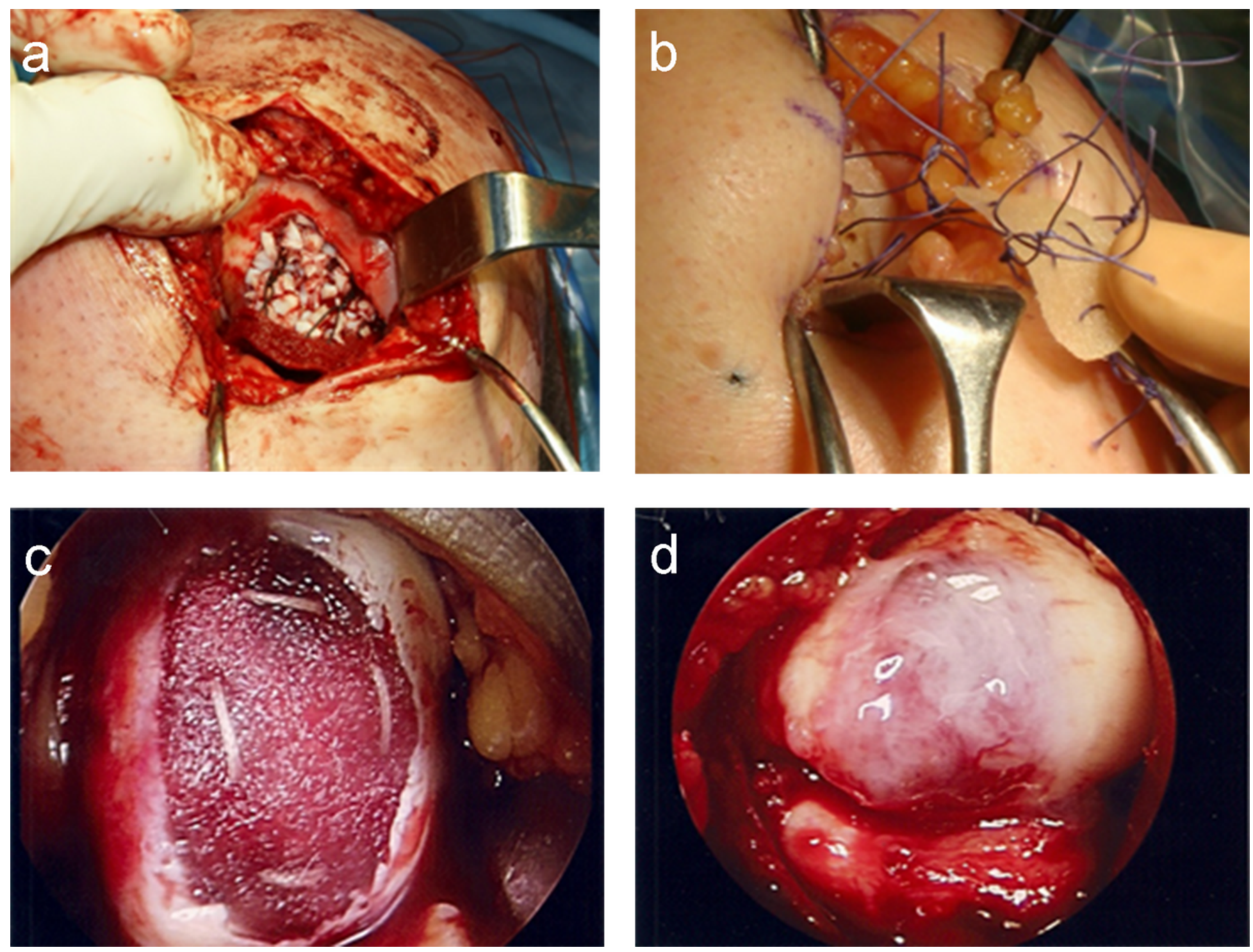

Figure 2

(a) Crushed autogenous cartilage. (b) Scaffold with transosseous sutures. (c) Scaffold in place. (d) Final appearance. 


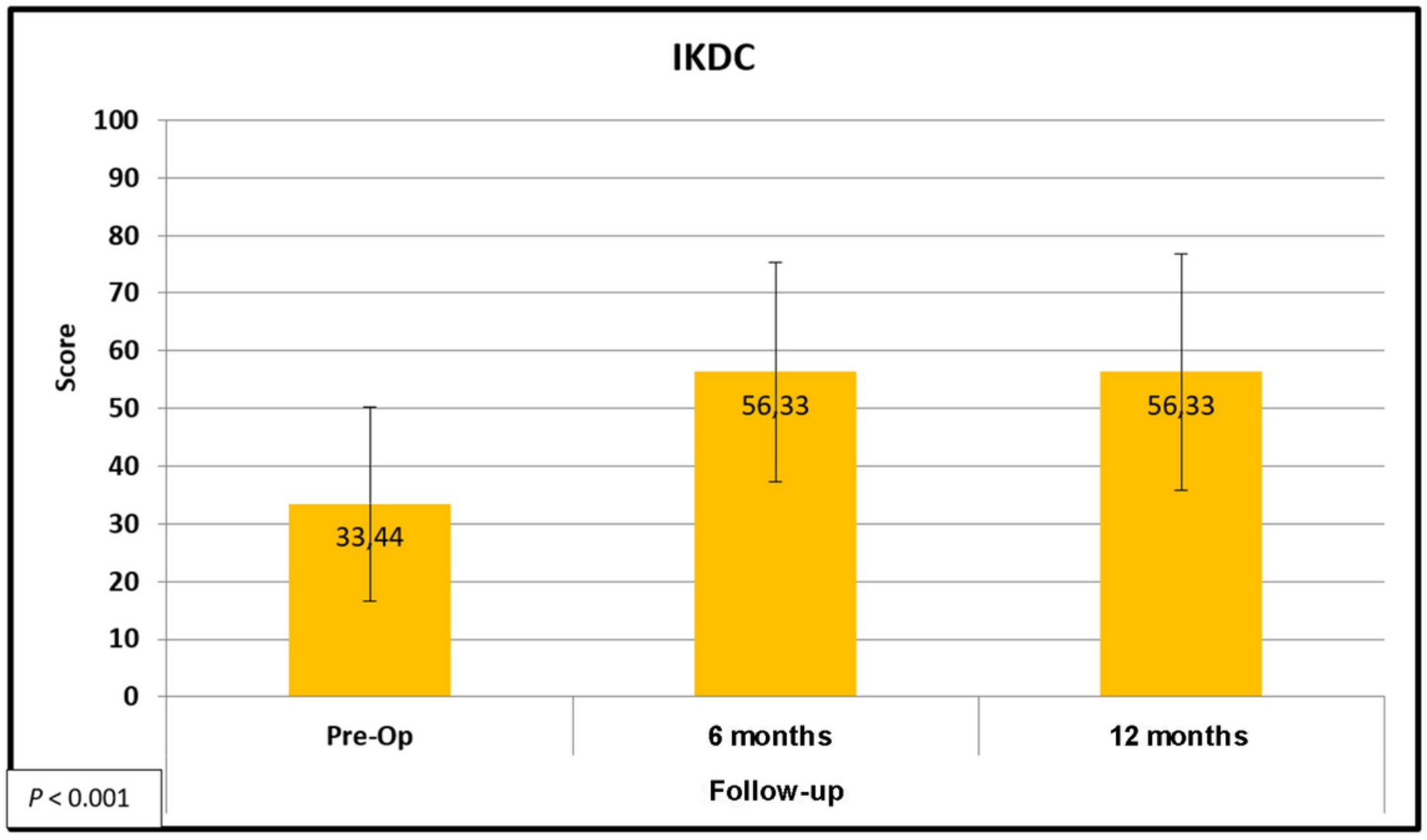

Figure 3

Descriptive clinical outcomes of articular joint repair: IKDC score. 


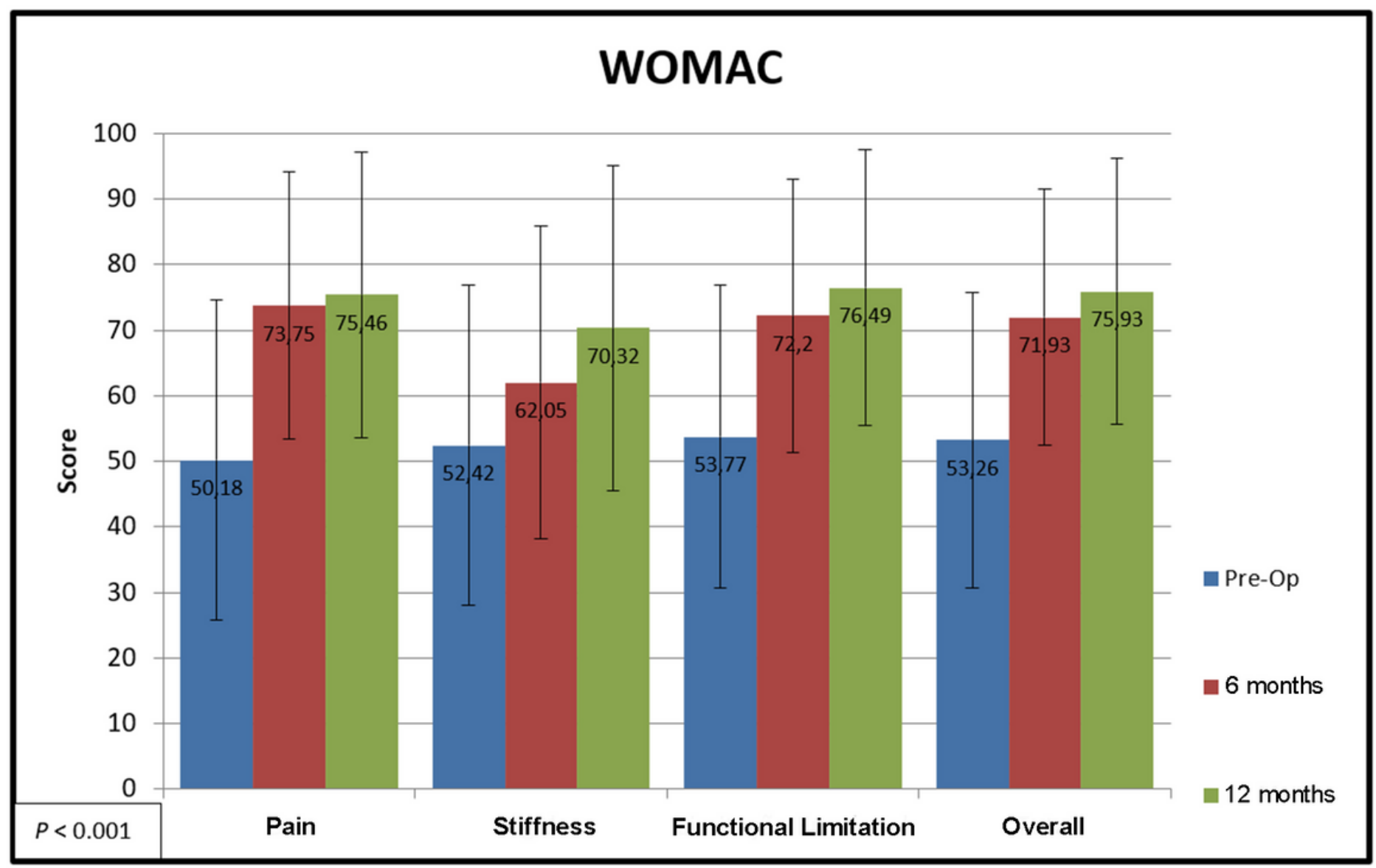

Figure 4

Descriptive clinical outcomes of articular joint repair: WOMAC score. 


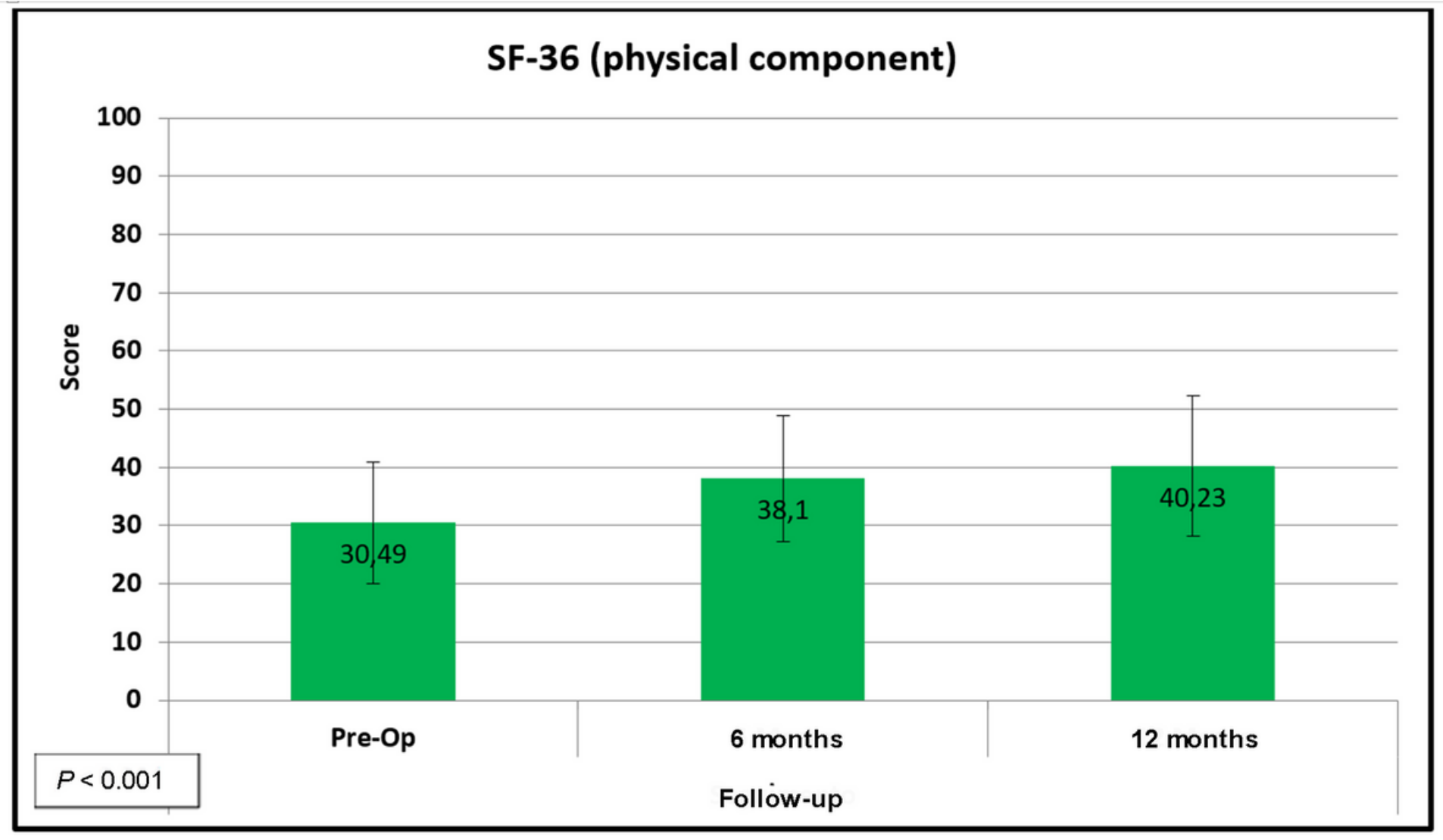

Figure 5

Descriptive clinical outcomes of articular joint repair: SF-36 score (physical domain). 